\title{
Dynamics of circle maps with flat spots
}

\author{
by \\ Jacek Graczyk (Orsay)
}

For M. Misiurewicz's 60th birthday

\begin{abstract}
We study a certain class of weakly order preserving, non-invertible circle maps with irrational rotation numbers and exactly one flat interval. For this class of circle maps we explain the geometric and dynamic structure of orbits. In particular, we formulate the so called upper and lower scaling rules which show an asymmetric and double exponential decay of geometry.
\end{abstract}

\section{Introduction}

1.1. Motivation. The objective of our presentation is to give a possibly complete description of the dynamics of a certain class of weakly order preserving, non-invertible circle maps which appear in a natural way (upper maps) while studying the dependence of the rotation interval on the parameter value for one-parameter families of endomorphisms of the circle (see [4]). A prototype family of this type is the sine family which constitutes one of the most famous models of transition to chaos:

$$
g_{t}(x)=x+(c / 2 \pi) \sin (2 \pi x)+t(\bmod 1) .
$$

Family (1) models a variety of physical phenomena and was studied intensively by mathematicians as well as physicists.

For $c \leq 1$ the maps $g_{t}$ are homeomorphisms of the circle to itself and the rotation number $\rho\left(g_{t}\right)$ of $g_{t}$ constitutes a topological invariant that measures the rate at which the orbit of a point wraps around the circle.

In general, for a weakly order preserving circle map the concept originated with Poincaré and is defined in terms of a lift $f$ to the real line as:

$$
\rho=\lim _{n \rightarrow \infty} \frac{f^{n}(x)}{n}
$$

2010 Mathematics Subject Classification: 37E10, 37E45.

Key words and phrases: circle map, flat interval, dynamical partition of the circle, nonwandering points, cross-ratio, right-ratio, scaling rule. 
where $x$ is any initial point. Both $f$ and the rotation number are defined uniquely up to shifts by integers.

When $c>1$ the maps $g_{t}$ are not invertible and have no rotation number (chaos). However, for these maps the notion of rotation interval can be introduced as a natural extension of the concept of rotation number for homeomorphisms [2]. There is an important result of [3] which says that the upper endpoint of the rotation interval is equal to the rotation number of a certain weakly order preserving circle map (upper map) which satisfies all our assumptions.

Assumptions and notation. We fix counterclockwise orientation of the circle. The metric on $S^{1}$ is given by the natural projection

$$
\Pi(x)=\exp (2 \pi i x)
$$

from the real line. Continuous circle maps can be lifted to the universal cover and written as continuous real maps obeying $f(x+1)-f(x) \in \mathbb{Z}$ for every real $x$. In this paper we will not make a strong distinction between objects defined on the circle and their lifts to the real line using both concepts according to our needs.

We denote by $|J|$ the length of the interval $J \subset S^{1}$ and by $\operatorname{dist}(x, y)$ or simply $|x-y|$ the length of the shortest closed interval which contains both $x$ and $y$.

The distance $\operatorname{dist}(X, Y)$ between two sets $X$ and $Y$ is defined as

$$
\inf \{\operatorname{dist}(x, y): x \in X, y \in Y\} .
$$

The left and the right endpoints of an interval $J$ will be denoted by $l(J)$ and $r(J)$ respectively.

Here is a list of the hypotheses.

HyPotheses.

1. We consider weakly order preserving circle maps $f$, at least twice continuously differentiable except for at most two points (endpoints of a flat interval).

2. The first derivative of $f$ is everywhere positive except for the closure of an open non-degenerate interval $I$ (the flat interval) on which it is equal to zero.

3. The right-sided derivative of $f$ at $r(I)$ is different from zero $(r(I)$ is not critical).

4. There exists a left-sided neighborhood of $l(I)$ and a real number $\ell>1$ so that for every $x$ from this neighborhood $f$ can be written as $f(x)=$ $-|\phi(x)|^{\ell}+f(I)$, where $\phi$ is a $C^{2}$ diffeomorphism on a neighborhood of $l(I)$.

5. The rotation number $\rho \in(0,1)$ of $f$ is irrational. 


\subsection{Preliminaries}

Uniform constants. We will mean by a uniform constant a function on our class of maps which depends continuously on the quasi-symmetric norm of the map on $S^{1} \backslash I$, the logarithm of the size of $I$, the order $\ell$ of the singularity of $f$ at $l(I)$, the $C^{2}$ norm of $f$ on $S^{1} \backslash I$ and the lower bound of the first derivative on $S^{1} \backslash(I \cup U)$, where $U$ (see Fact 2.1 in the next subsection) is a left-sided neighborhood of $l(I)$ on which the second derivative is negative. Uniform positive constants will be usually denoted by the letters $K, A, M$ with suitable subscripts, and we always assume $0<\lambda<1$.

Superexponential convergence. In our estimates we will often use a convergence which is faster than exponential. Typically, it will be double exponential convergence given by sequences of the form $\exp (-\exp (K n))$ where $K$ is a uniform constant.

Definition 1.1. We say that a sequence of real numbers $c_{n}$ tends to $c \in \mathbb{R}$ uniformly superexponentially if there exist uniform positive constants $M$ and $K$ such that for every $n \geq M$,

$$
\left|c_{n}-c\right| \leq \exp (-\exp (K n)) \text {. }
$$

Continued fractions and dynamics. The rotation number $\rho$ can be written as an infinite continued fraction

$$
\rho=\frac{1}{a_{1}+\frac{1}{a_{2}+\frac{1}{\ldots}}},
$$

where $a_{i}$ are positive integers. If we cut off the portion of the continued fraction beyond the $n$th position, and write the resulting fraction in lowest terms as $p_{n} / q_{n}$ then the numbers $q_{n}$ for $n \geq 1$ satisfy the recurrence relation

$$
q_{n+1}=a_{n+1} q_{n}+q_{n-1}, \quad q_{0}=1, q_{1}=a_{1} .
$$

The number $q_{n}$ is the iterate of the rotation by $\rho$ for which the orbit of any point makes the closest return so far to the point itself.

By the Poincaré Theorem, maps from our class are semi-conjugate to a rotation. In particular, this implies the same order of orbits for both $f$ and the rotation by $\rho$. The numbers $q_{n}$ are called closest returns.

Continued fractions and partitions. We will use the preimages of the flat interval $I$ to define a system of partitions of the circle.

Consider all $q_{n}+q_{n+1}-1$ preimages of $I$ and $I$ itself together with all holes between successive preimages of $I$. Set, for $n$ even,

$$
\square_{i}^{n}=f^{-i}\left(r\left(f^{-q_{n}}(I)\right), l(I)\right) \quad \text { and } \square_{i}^{n+1}=f^{-i}\left(r(I), l\left(f^{-q_{n+1}}(I)\right)\right),
$$

and for $n$ odd,

$$
\square_{i}^{n}=f^{-i}\left(r(I), l\left(f^{-q_{n}}(I)\right)\right) \quad \text { and } \quad \square_{i}^{n+1}=f^{-i}\left(r\left(f^{-q_{n+1}}(I)\right), l(I)\right) .
$$


Thus, all holes of the $n$th partition can be divided into two groups:

- $q_{n+1}$ "long" holes $\square_{0}^{n}, \ldots, \square_{q_{n+1}-1}^{n}$,

- $q_{n}$ "short" holes $\square_{0}^{n+1}, \ldots, \square_{q_{n}-1}^{n+1}$.

The partition obtained by the above construction will be denoted by $\mathcal{A}_{n}$ and called the $n$th dynamical partition of the circle.

We will briefly explain the structure of the partitions. Take two subsequent dynamical partitions of order $n$ and $n+1$. The latter is clearly a refinement of the former. All "short" holes of $\mathcal{A}_{n}$ become "long" holes of $\mathcal{A}_{n+1}$ while all "long" holes of $\mathcal{A}_{n}$ split into $a_{n+2}$ preimages of $I$ and $a_{n+2}$ "long" holes and one "short" hole of the next partition $\mathcal{A}_{n+1}$ :

$$
\square_{i}^{n}=\bigcup_{j=1}^{a_{n+2}} f^{-i-q_{n}-j q_{n+1}}(I) \cup \bigcup_{j=0}^{a_{n+2}-1} \square_{i+q_{n}+j q_{n+1}}^{n+1} \cup \square_{i}^{n+2} .
$$

Several of the proofs in the following will strongly depend on the relative positions of the points and intervals of the dynamical partitions. When reading the proofs the reader is advised to keep the following picture in mind, which shows some of these objects near the flat interval $I$. To denote the relevant objects in the picture we use temporary notation: $\underline{i}$ stands for $f^{i}(I)$ and $\underline{0}$ stands for $I$.

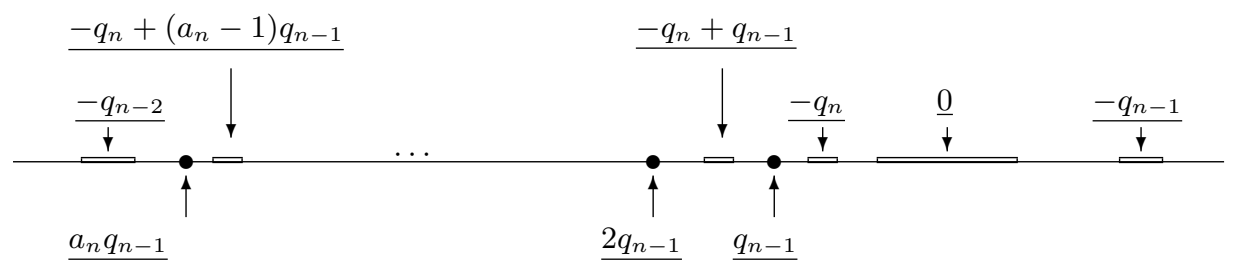

REMARK. Observe that $f^{q_{n}}(I)$ lies to the right of $I$ for $n$ even, and to the left of $I$ for $n$ odd. If we do not want to specify holes of the $n$th partition then we denote them briefly by $\square(n)$.

\subsection{Presentation of results}

Description and statement of results. We begin by recalling the definition of the set $\Omega$.

DeFinition 1.2. A point $x$ belongs to the set of non-wandering points $\Omega(f)$ if and only if for any open neighborhood $V \ni x$ there exists an integer $n>0$ such that the intersection of $V$ and $f^{n}(V)$ is non-empty, where $f^{n}$ stands for the $n$th composition of $f$.

Our goal is to describe both the behavior of high iterates of the map $f$ and the geometric structure of the set $\Omega$ of non-wandering points.

Upper scaling rules. The crucial technical observation is stated as the First Basic Lemma (FBL) below. The scaling rules formulated there, by 
which we mean the ratio of the distance between the flat interval and its two successive closest returns from the same side, give the upper bounds of the geometry of the Cantor set $\Omega$. We prove that the scaling rules are at least superexponentially decreasing and do not depend on even coefficients in the continued fraction expansion of the rotation number. This reflects the hybrid dynamics of $f$ which is topologically expansive, i.e. maps a proper interval $\left(S^{1} \backslash I\right)$ onto the whole circle, critical of polynomial type at the left side of $I$, and non-degenerate at the right side of $I$.

Let $p_{n} / q_{n}$ be the $n$th convergent to the continued fraction representation of the rotation number $\rho=\left[0, a_{1}, \ldots, a_{n}, \ldots\right]$. Put $\gamma(1)=1$ and for all $n>1$,

$$
\gamma(n):=\gamma(\rho, n)=\prod_{0<i \leq[n / 2]}\left(1+\frac{a_{2 i+1}}{\ell}\right) .
$$

Lemma 1.1 (First Basic Lemma). Suppose that $f$ satisfies the Hypotheses. There is a uniform constant $P>0$ and a constant $0<\lambda<1$ such that for every $n \geq 1$ :

$$
\operatorname{dist}\left(f^{q_{n}}(I), I\right) \leq \lambda^{\gamma(n)}, \quad \frac{\left|f^{-q_{n}}(I)\right|}{\operatorname{dist}\left(f^{-q_{n}}(I), I\right)} \geq P \lambda^{-\gamma(n)} .
$$

Further, there exist uniform constants $N, A>0$ such that for every integer $n \geq N$, the constant $\lambda$ can be chosen as $\left(\operatorname{dist}\left(f^{q_{N}}(I), I\right)\right)^{A / \gamma(N)}$.

A uniform version of FBL is stated in Section 4. As an immediate corollary of the upper scaling rules we obtain Theorem 1 which says that independently of the rotation number the flat interval $I$ is approximated at least superexponentially by successive closest returns $f^{q_{n}}(I)$.

THEOREM 1. If $f$ satisfies the Hypotheses then there is a positive constant $0<\lambda<1$ such that $\operatorname{dist}\left(f^{q_{n}}(I), I\right)$ tends to zero faster than $\lambda^{(\sqrt{1+1 / \ell})^{n}}$.

Lower scaling rules. The upper scaling rules allow us to formulate distortion and convexity lemmas in Sections 5 and 6. These analytic estimates lead to the Second Basic Lemma (SBL) setting lower bounds for scaling rules.

Put $\bar{\gamma}(1)=1$ and for every $n>1$ define

$$
\bar{\gamma}(n):=\bar{\gamma}(\rho, n)=\prod_{0<i \leq[n / 2]}\left(1+\frac{\ell+1}{\ell-1} a_{2 i+1}\right) .
$$

Lemma 1.2 (Second Basic Lemma). Let $f$ satisfy the Hypotheses. There is a uniform constant $N$ and a constant $0<\lambda<1$ such that for every $n \geq N$,

$$
\operatorname{dist}\left(f^{q_{n}}(I), I\right) \geq \lambda^{\bar{\gamma}(n)}, \quad \frac{\left|f^{-q_{n}}(I)\right|}{\operatorname{dist}\left(f^{-q_{n}}(I), I\right)} \leq \lambda^{-\bar{\gamma}(n)} .
$$

The constant $\lambda$ can be chosen as $\left(\operatorname{dist}\left(f^{q_{N}}(I), I\right)\right)^{A / \bar{\gamma}(N)}$, where $A$ is a uniform constant. 
REMARKS. Similar but somewhat different scaling rules were considered in [5] for rotation numbers of bounded type; that is, where all coefficients $a_{i}$ of the continued fraction expansion of $\rho$ are supposed to be bounded. A further study of scaling rules for circle maps with a flat interval and rotation numbers of bounded type can be found in [1].

Scaling rules of Fact 3.3 below imply that the set $\Omega$ of non-wandering points coincides with $S^{1} \backslash \bigcup_{n=0}^{\infty} f^{-n}(I)$ and is of zero Lebesgue measure for every map satisfying the Hypotheses (Proposition 1). For rotation numbers of bounded type, the Hausdorff dimension of $\Omega$ was studied in [1].

2. Technical tools. Our main technical tools are right-ratio and crossratio inequalities.

The cross-ratio inequality (CRI). We shall adapt the cross-ratio inequality introduced in [4] to our needs. For four points $a, b, c, d$ arranged according to the standard orientation of the circle so that $a<b<c<d$ and $b, c \in(a, d)$ we define their cross-ratio as

$$
\mathbf{C r}(a, b, c, d)=\frac{|a-b||c-d|}{|a-c||b-d|} .
$$

By the distortion of the cross-ratio we mean

$$
\mathcal{D} \mathbf{C r}(a, b, c, d)=\frac{\mathbf{C r}(f(a), f(b), f(c), f(d))}{\mathbf{C r}(a, b, c, d)} .
$$

Let us consider a set of quadruples $\left\{a_{i}, b_{i}, c_{i}, d_{i}\right\}$ with the following properties:

1. Each point of the circle belongs to at most $k$ intervals $\left(a_{i}, d_{i}\right)$.

2. The intervals $\left(b_{i}, c_{i}\right)$ do not intersect $I$. Then

$$
\prod_{i=0}^{n} \mathcal{D} \mathbf{C r}\left(a_{i}, b_{i}, c_{i}, d_{i}\right) \leq C_{k}
$$

and the constant $C_{k}$ does not depend on the set of quadruples.

The right-ratio inequality (RRI). Suppose we have three points $a, b, c$ so that $a<b<c$. The right-ratio and its distortion are defined as follows:

$$
\mathbf{R}(a, b, c)=\frac{|b-c|}{|a-b|}, \quad \mathcal{D} \mathbf{R}(a, b, c)=\frac{\mathbf{R}(f(a), f(b), f(c)}{\mathbf{R}(a, b, c)} .
$$

Consider now a set of triples $\left\{a_{i}, b_{i}, c_{i}\right\}, 0 \leq i \leq n$, which satisfy the following conditions:

1. Each point of the circle belongs to at most $k$ intervals $(a, c)$.

2. The intervals $\left(a_{i}, b_{i}\right)$ do not intersect the flat interval $I$ and $I$ is not contained in $\left(b_{i}, c_{i}\right)$. 
Then

$$
\prod_{i=0}^{n} \mathcal{D} \mathbf{R}\left(a_{i}, b_{i}, c_{i}\right) \leq R_{k}
$$

and the constant $R_{k}$ does not depend on the set of triples.

REMARK. In this paper we will consider only sets of triples and quadruples formed by taking iterations either of an initial triple or quadruple. Therefore, to characterize a given set of triples or quadruples we will indicate the initial object in braces followed after a semicolon by the number of iterates one must perform to get all of them.

Proof of the right-ratio inequality. We start with a simple fact from calculus.

FACT 2.1. There is a left-sided neighborhood $U$ of the point $l(I)$ such that for all $y \in U$ the second derivative of $f$ is negative. We can also assume that $|U|<1 / 2$ is so small that $|f(U)|<1 / 2$.

Next we list some properties of the right-ratio which immediately yield the desired result.

1. Upper convexity of $f$ restricted to $U$ implies that the distortion of the right-ratio is strictly less than 1 for triples contained in $U \cup I$.

2. Let $U^{\prime}$ be a left-sided neighborhood of $l(I)$ strictly contained in $U$. A total distortion of the right-ratio for triples contained in the complement of $I \cup U^{\prime}$ is bounded by the variation of $\log \frac{d f}{d x}$ over this set.

3. There are at most $k$ triples which are not covered by any of the above two cases. For these triples the distortion of the right-ratio is also bounded, as can be checked by a direct calculation.

3. Topological description of the set $\Omega$. There is the following characterization of the set $\Omega$.

Proposition 1. The set $\Omega$ is equal to $S^{1} \backslash \bigcup_{i=0}^{\infty} f^{-i}(I)$ and is of zero Lebesgue measure.

We start the proof of Proposition 1 with two simple observations. Let $U$ be the neighborhood from Fact 2.1.

FACT 3.1. There are constants $K_{1}, K_{2}$ such that if $y \in U$ then

$$
\begin{gathered}
K_{1}|l(I)-y|^{\ell} \leq|f(l(I))-f(y)| \leq K_{2}|l(I)-y|^{\ell}, \\
K_{1}|l(I)-y|^{\ell-1} \leq \frac{d f}{d x}(y) \leq K_{2}|l(I)-y|^{\ell-1} .
\end{gathered}
$$

FACT 3.2. There is a constant $K_{3}$ such that for every $y, z \in U$,

provided $z \in(y, l(I))$.

$$
\frac{|f(y)-f(z)|}{|f(y)-f(I)|} \leq K_{3} \frac{|y-z|}{|y-l(I)|}
$$


LEMma 3.1. There is a uniform constant $K_{4}>0$ such that

$$
\frac{\left|f^{-q_{n}}(I)\right|}{\operatorname{dist}\left(f^{-q_{n}}(I), I\right)} \geq K_{4} \text {. }
$$

The sequence $\operatorname{dist}\left(f^{q_{n}}(I), I\right)$ tends to zero uniformly and at least exponentially.

Proof. First, we prove that the length of $f^{-q_{n}}(I)$ cannot be too small in comparison to the distance to the flat interval $I$. Assume that $n$ is even, i.e. $f^{-q_{n}}(I)$ is to the left of $I$.

Apply CRI to

$$
\left\{l\left(f^{-q_{n}+1}(I)\right), r\left(f^{-q_{n}+1}(I)\right), l\left(f^{-q_{n+1}+1}(I)\right), r\left(f^{-q_{n+1}+1}(I)\right) ; q_{n}-1\right\}
$$

to obtain

$$
\begin{aligned}
& \frac{|I|}{\left|l(I)-l\left(f^{-q_{n+1}+q_{n}}(I)\right)\right|} \frac{\left|f^{-q_{n+1}+q_{n}}(I)\right|}{\left|r(I)-r\left(f^{-q_{n+1}+q_{n}}(I)\right)\right|} \\
\leq & C_{1} \frac{\left|f^{-q_{n}+1}(I)\right|}{\left|l\left(f^{-q_{n}+1}(I)\right)-l\left(f^{-q_{n+1}+1}(I)\right)\right|} \frac{\left|f^{-q_{n+1}+1}(I)\right|}{\left|r\left(f^{-q_{n}+1}(I)\right)-r\left(f^{-q_{n+1}+1}(I)\right)\right|} .
\end{aligned}
$$

Fact 3.2 yields

$$
\frac{\left|f^{-q_{n}+1}(I)\right|}{\left|l\left(f^{-q_{n}+1}(I)\right)-f(I)\right|} \leq K_{3} \frac{\left|f^{-q_{n}}(I)\right|}{\left|l\left(f^{-q_{n}}(I)\right)-l(I)\right|} .
$$

The first factor of the left-hand side of $(2)$ is bounded away from zero while the second factor on the right-hand side is less than 1. Therefore,

$$
\frac{\left|f^{-q_{n}}(I)\right|}{\left|l\left(f^{-q_{n}}(I)\right)-l(I)\right|} \geq K_{5} \frac{\left|f^{-q_{n+1}+q_{n}}(I)\right|}{\left|r(I)-r\left(f^{-q_{n+1}+q_{n}}(I)\right)\right|} .
$$

The interval $f^{-q_{n+1}+q_{n}}(I)$ lies between $r(I)$ and $r\left(f^{-q_{n+1}+q_{n}}(I)\right)$. Therefore, RRI written for $\left\{r(I), l\left(f^{-q_{n+1}+q_{n}}(I)\right), r\left(f^{-q_{n+1}+q_{n}}(I)\right) ; q_{n+1}-q_{n}\right\}$ gives a lower bound of (3), which yields the inequality from Lemma 3.1.

For $n$ odd, the considerations are much simpler. To get the same estimates as above it is enough to write $\mathbf{R R I}$ for $\left\{r(I), l\left(f^{-q_{n}}(I)\right), r\left(f^{-q_{n}}(I)\right) ; q_{n}\right\}$.

Finally for both cases, since $f^{q_{n+1}}(I)$ lies between $f^{-q_{n}}(I)$ and $I$ we obtain

$$
\operatorname{dist}\left(f^{q_{n+1}}(I), I\right) \leq\left(1 /\left(1+K_{4}\right)\right) \operatorname{dist}\left(f^{q_{n-1}}(I), I\right),
$$

which completes the proof of Lemma 3.1 .

First estimates. We write down four simple inequalities which will set a basis for the proof of the main estimates in the next section. We will also use them to prove Proposition 1 .

Consider the $n$th partition of the circle. We form a set of initial triples by taking the endpoints of all holes $\square(n) \in \mathcal{A}_{n}$ together with the right endpoint 
of the preimage $I(\square(n)) \in \mathcal{A}_{n}$ of the flat interval $I$ lying immediately to the right of $\square(n) \in \mathcal{A}_{n}$. RRI implies:

FACT 3.3. The ratio

$$
\frac{|\square(n)|}{|I(\square(n))|}
$$

is bounded from above by either

- $\left.\left(R_{1} /|I|\right) \operatorname{dist}\left(f^{q_{n}}(I), I\right)\right)$ or $\left.\left(R_{1} /|I|\right) \operatorname{dist}\left(f^{-q_{n+1}}(I), I\right)\right)$ for $n$ odd,

- $\left.\left(R_{1} /|I|\right) \operatorname{dist}\left(f^{-q_{n}}(I), I\right)\right)$ or $\left.\left(R_{1} /|I|\right) \operatorname{dist}\left(f^{q_{n+1}}(I), I\right)\right)$ for $n$ even.

Proof of Proposition 1. The inclusion $\Omega \subset S^{1} \backslash \bigcup_{i=0}^{\infty} f^{-i}(I)$ is evident. So, we have to prove that each point in $S^{1} \backslash \bigcup_{i=0}^{\infty} f^{-i}(I)$ is non-wandering. By Fact 3.3 the complement of all preimages of $I$ does not contain any density point with respect to the Lebesgue measure. Hence, by the Lebesgue Density Lemma the set of non-wandering points is of zero Lebesgue measure. In particular, $\bigcup_{i=0}^{\infty} f^{-i}(I)$ is dense in $S^{1}$. Moreover, $S^{1} \backslash \bigcup_{i=0}^{\infty} f^{-i}(I)$ contains no isolated points, because such an isolated point would lie on the boundary of two inverse images of $I$ and therefore would imply the existence of a periodic point. Since $f$ is order preserving, we see that $\left\{f^{i}(I): i>0\right\}$ is dense in $S^{1} \backslash \bigcup_{i=0}^{\infty} f^{-i}(I)$. Hence, every point in $S^{1} \backslash \bigcup_{i=0}^{\infty} f^{-i}(I)$ has a dense orbit, by the property of $f$ being order preserving.

4. Scaling rules-upper bounds. We recall that $p_{n} / q_{n}$ is the $n$th convergent of the continued fraction representation of the rotation number $\rho=\left[0, a_{1}, \ldots, a_{n}, \ldots\right]$.

Let $1 \leq m<n$ and define

$$
\gamma(n, m):=\gamma(\rho, n, m)=\prod_{[m / 2]<i \leq[n / 2]}\left(1+\frac{a_{2 i+1}}{\ell}\right) .
$$

In the above definition, we adopt the rule that the product over an empty set of indices is equal to 1 . For example, if $n$ is odd then $\gamma(n, n-1)=1$. Also, $\gamma(n, n)=1$. We see that $\gamma(n)=\gamma(n, 1)$. By the definition, for any $m \leq k \leq n, \gamma(n, m)=\gamma(n, k) \gamma(k, m)$.

Our main technical task in this section is to prove FBL.

We want to compare the length of $f^{-q_{n}}(I)$ with $\operatorname{dist}\left(f^{-q_{n}}(I), I\right)$ and show that the latter is much smaller than the former. Our considerations fall into two parts:

\subsection{Proof of FBL when $f^{-q_{n}}(I)$ is to the left of $I$}

LEMmA 4.1. There are uniform constants $n_{0}, K_{6}$ such that

$$
\left|r(I)-l\left(f^{-q_{n-1}}(I)\right)\right|^{1 / \ell} \leq K_{6}\left|f^{-q_{n}}(I)\right| \quad \text { for all } n \geq n_{0} .
$$

Proof. From Lemma 3.1 there is $n_{0}$ such that for every $n \geq n_{0}$ even, $f^{-q_{n-2}}(I) \subset U$, where $U$ is the neighborhood from Fact 2.1 . 
We will show that the opposite inequality

$$
\left|r(I)-l\left(f^{-q_{n-1}}(I)\right)\right|^{1 / \ell} \geq(1 / \alpha)\left|f^{-q_{n}}(I)\right|
$$

leads to a contradiction if only $\alpha$ is small enough.

Since the right-sided derivative of $f$ at $r(I)$ is positive, the lengths $\left|f(I)-l\left(f^{-q_{n-1}+1}(I)\right)\right|$ and $\left|r(I)-l\left(f^{-q_{n-1}}(I)\right)\right|$ are comparable. By the inequality (4) we get

$$
\left|f(I)-l\left(f^{-q_{n-1}+1}(I)\right)\right|^{1 / \ell} \geq\left(K_{7} / \alpha\right)\left|f^{-q_{n}}(I)\right|
$$

and consequently, by Lemma 3.1 and Fact 3.1 .

$$
\geq\left(K_{8} / \alpha\right)\left|l\left(f^{-q_{n}+1}(I)\right)-f(I)\right|^{1 / \ell} .
$$

Thus

$$
\left|f(I)-l\left(f^{-q_{n-1}+1}(I)\right)\right| \geq\left(K_{8} / \alpha\right)^{\ell}\left|l\left(f^{-q_{n}+1}(I)\right)-f(I)\right| .
$$

For $a_{n} \geq i \geq 0$ set

$$
\beta_{i}=\left|l\left(f^{-q_{n}+i q_{n-1}}(I)\right)-f^{i q_{n-1}}(I)\right|, \quad S_{i}=\left|l\left(f^{-q_{n}+i q_{n-1}}(I)\right)-l(I)\right| .
$$

We shall show that

$$
\frac{\beta_{i+1}}{S_{i+1}} \leq\left(K_{9} \alpha \frac{S_{i}}{\beta_{0}}\right)^{\ell} \frac{\beta_{i}}{S_{i}}
$$

for all $0 \leq i \leq a_{n}-1$.

Writing CRI for

$$
\left\{l\left(f^{-q_{n}+i q_{n-1}+1}(I)\right), f^{i q_{n-1}+1}(I), l\left(f^{-q_{n-1}+1}(I)\right), r\left(f^{-q_{n-1}+1}(I)\right) ; q_{n-1}-1\right\},
$$

we obtain

$$
\frac{|I|}{\mid f^{(i+1) q_{n-1}(I)-r(I) \mid}} \frac{\beta_{i+1}}{S_{i+1}}
$$

$$
\leq C_{1} \frac{\left|l\left(f^{-q_{n}+i q_{n-1}+1}(I)\right)-f^{i q_{n-1}+1}(I)\right|}{\left|l\left(f^{-q_{n}+i q_{n-1}+1}(I)\right)-l\left(f^{-q_{n-1}+1}(I)\right)\right|} \frac{\left|f^{-q_{n-1}+1}(I)\right|}{\left|f^{i q_{n-1}+1}(I)-r\left(f^{-q_{n-1}+1}(I)\right)\right|} .
$$

By Facts 3.2 and 3.1 we have

$$
\begin{gathered}
\frac{\left|l\left(f^{-q_{n}+i q_{n-1}+1}(I)\right)-f^{i q_{n-1}+1}(I)\right|}{\left|l\left(f^{-q_{n}+i q_{n-1}+1}(I)\right)-f(I)\right|} \leq K_{3} \frac{\beta_{i}}{S_{i}}, \\
\frac{\left|l\left(f^{-q_{n}+i q_{n-1}+1}(I)\right)-f(I)\right|}{\left|l\left(f^{-q_{n}+1}(I)\right)-f(I)\right|} \leq\left(K_{2} / K_{1}\right)\left(\frac{S_{i}}{\beta_{0}}\right)^{\ell} .
\end{gathered}
$$

The first factor on the left-hand side of (7) is greater than a uniform constant. We can also neglect the last factor on the right-hand side of (7) as it is smaller than 1. Further, we substitute (8) and (9) into the resulting inequality to obtain

$$
\frac{\beta_{i+1}}{S_{i+1}} \leq K_{10}\left(\frac{S_{i}}{\beta_{0}}\right)^{\ell} \frac{\left|f(I)-l\left(f^{-q_{n}+1}(I)\right)\right|}{\left|l\left(f^{-q_{n}+i q_{n-1}+1}(I)\right)-l\left(f^{-q_{n-1}+1}(I)\right)\right|} \frac{\beta_{i}}{S_{i}} .
$$


Note that

$$
\left(f(I), l\left(f^{-q_{n-1}+1}(I)\right)\right) \subset\left(l\left(f^{-q_{n}+i q_{n-1}+1}(I)\right), l\left(f^{-q_{n-1}+1}(I)\right)\right) .
$$

Therefore, substituting (5) into (10) we finally obtain (6).

We pass to estimating the rate of growth of $\beta_{i} / S_{i}$. To simplify the notation we set new $\alpha$ to be equal to $K_{9} \alpha$.

We begin by showing that $S_{i+1}-S_{i} \leq \kappa \beta_{i+1}$ for a uniform constant $\kappa>1$. To do this we define $\epsilon_{i+1}=\left|f^{(i+1) q_{n-1}}(I)-l\left(f^{-q_{n}+i q_{n-1}}(I)\right)\right|$. Observe that $S_{i+1}-S_{i}=\beta_{i+1}+\epsilon_{i+1}$. The interval $\left(f^{(i+1) q_{n-1}}(I), l\left(f^{-q_{n}+i q_{n-1}}(I)\right)\right)$ is contained in the hole $\left(r\left(f^{-q_{n}+(i+1) q_{n-1}}(I)\right), l\left(f^{-q_{n}+i q_{n-1}}(I)\right)\right)$ of the dynamical partition $\mathcal{A}_{n-1}$, and therefore the error term $\epsilon_{i+1}$ can be estimated using the cross-ratio inequality written for the endpoints of the intervals $f^{-q_{n}+(i+1) q_{n-1}}(I)$ and $f^{-q_{n}+i q_{n-1}}(I)$ and the number of iterates $q_{n}-$ $(i+1) q_{n-1}$ (see the figure below with a temporary notation $\underline{i}:=f^{i}(I)$ and $\underline{0}:=I)$.

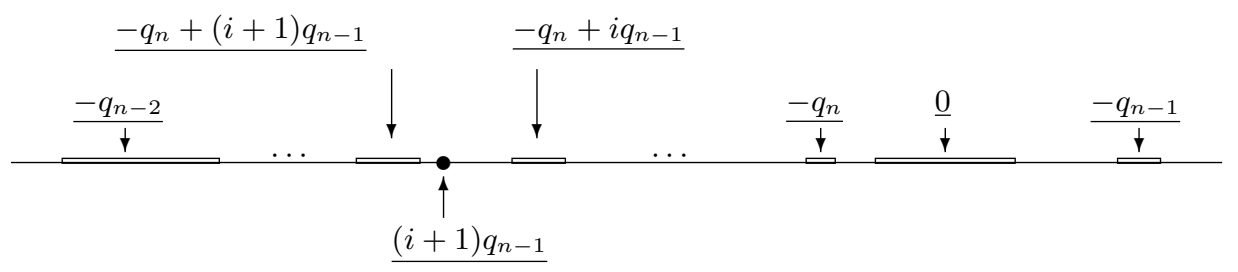

Indeed, using simple algebra and Lemma 3.1, we have

$$
\begin{aligned}
& \frac{\beta_{i+1}}{\epsilon_{i+1}+\beta_{i+1}} \geq \frac{1}{C_{1}} \frac{|I|}{\left|l(I)-l\left(f^{-q_{n-1}}(I)\right)\right|} \frac{\left|f^{-q_{n-1}}(I)\right|}{\mid r(I)-r\left(f^{-q_{n-1}}(I) \mid\right.} \\
& \geq \frac{K_{4}|I|}{C_{1}\left(1+K_{4}\right)} \text {. }
\end{aligned}
$$

In particular, there exists a uniform constant $\kappa>1$ such that $\beta_{i+1}+\epsilon_{i+1}$ $\leq \kappa \beta_{i+1}$ for every $0 \leq i \leq a_{n}-1$.

We shall show that for all $0 \leq i \leq a_{n}$ the following inequalities are true if only $\alpha$ is small enough:

(i) $S_{i} \leq \beta_{0}\left(1+2 \alpha^{\ell-1}\right)\left(1+\alpha^{\ell-1}\right)\left(1+\alpha^{\ell-1} / 2\right) \cdots\left(1+\alpha^{\ell-1} / 2^{i-1}\right)$,

(ii) $\beta_{i} / S_{i} \leq \alpha^{i(l-1)}$.

Induction. For $i=0$ our inequalities trivially hold. Suppose that the induction hypothesis is true for some $i \leq a_{n}-1$ and $\alpha$ is chosen so that $\alpha \exp \left(4 \ell \alpha^{\ell-1}\right) \leq 1$. Further, using (6) with the new $\alpha$, we obtain

$$
\beta_{i+1} / S_{i+1} \leq\left(\alpha \exp \left(4 \alpha^{\ell-1}\right)\right)^{\ell}\left(\beta_{i} / S_{i}\right) \leq \alpha^{\ell-1}\left(\beta_{i} / S_{i}\right) \leq \alpha^{(\ell-1)(i+1)} .
$$

To prove the first inequality we assume additionally that $\alpha^{\ell-1}<1 / 4 \kappa$ and use the fact that the function $x \mapsto x /(1-x)$ is increasing for $x<1$. 
Then

$$
\begin{aligned}
S_{i+1} & =S_{i}+\beta_{i+1}+\epsilon_{i+1} \leq\left(1+\frac{\left(\epsilon_{i+1}+\beta_{i+1}\right) / S_{i+1}}{1-\left(\epsilon_{i+1}+\beta_{i+1}\right) / S_{i+1}}\right) S_{i} \\
& =\left(1+\frac{\kappa \alpha^{(i+1)(\ell-1)}}{1-\kappa \alpha^{(i+1)(\ell-1)}}\right) S_{i} \leq\left(1+\frac{\kappa \alpha^{i(\ell-1)}}{1-\kappa \alpha^{i(\ell-1)}} \alpha^{\ell-1}\right) S_{i} \\
& \leq \beta_{0}\left(1+2 \alpha^{\ell-1}\right) \cdots\left(1+\alpha^{\ell-1} / 2^{i}\right) .
\end{aligned}
$$

Conclusion of the proof of Lemma 4.1. As a consequence of (ii), we obtain

$$
\beta_{a_{n}} / S_{a_{n}} \leq \alpha^{(\ell-1) a_{n}} .
$$

Let us recall that $-q_{n-2}=-q_{n}+a_{n} q_{n-1}$. Fact 3.2 and $\mathbf{C R I}$ written for

$$
\left\{l\left(f^{-q_{n-2}+1}(I)\right), f^{a_{n} q_{n-1}+1}(I), l\left(f^{-q_{n-1}+1}(I)\right), r\left(f^{-q_{n-1}+1}(I)\right) ; q_{n-2}-1\right\}
$$

imply that

$$
\begin{aligned}
\frac{\beta_{a_{n}}}{S_{a_{n}}} & \geq \frac{1}{K_{3}} \frac{\left|l\left(f^{-q_{n-2}+1}(I)\right)-f^{a_{n} q_{n-1}+1}(I)\right|}{\left|l\left(f^{-q_{n-2}+1}(I)\right)-l\left(f^{-q_{n-1}+1}(I)\right)\right|} \\
& \geq \frac{|I|}{C_{1} K_{3}} \frac{\left|f^{-q_{n-1}+q_{n-2}}(I)\right|}{\left|f^{q_{n}}(I)-r\left(f^{-q_{n-1}+q_{n-2}}(I)\right)\right|} .
\end{aligned}
$$

By RRI, the right-hand side of the above inequality is bounded from below by a constant $K_{11}$. Choosing $\alpha^{\ell-1}$ smaller than $|I| K_{11} / C_{1} K_{1}$, we obtain a contradiction.

Conclusion of the proof of FBL. By Lemma 4.1 and Fact 3.1 we obtain

$$
\frac{\left|f^{-q_{n}}(I)\right|}{\left.\mid r\left(f^{-q_{n}}(I)\right)-l(I)\right) \mid} \geq K_{12}\left(\frac{\left|f(I)-l\left(f^{-q_{n-1}+1}(I)\right)\right|}{\left|r\left(f^{-q_{n}+1}(I)\right)-f(I)\right|}\right)^{1 / \ell} .
$$

Let $0 \leq i \leq a_{n+1}-2$ (we temporarily assume that $a_{n+1}>1$ ). RRI written for

$$
\left\{f(I), l\left(f^{-q_{n-1}-(i+1) q_{n}+1}(I)\right), l\left(f^{-q_{n-1}-i q_{n}+1}(I)\right) ; q_{n-1}+(i+1) q_{n}-1\right\}
$$

yields

$$
\frac{\left|f(I)-l\left(f^{-q_{n-1}-(i+1) q_{n}+1}(I)\right)\right|}{\left|f(I)-l\left(f^{-q_{n-1}-i q_{n}+1}(I)\right)\right|} \leq\left(R_{1} /|I|\right) \operatorname{dist}\left(f^{q_{n-1}}(I), I\right) .
$$

Multiply through the inequalities (12) for $0 \leq i \leq a_{n+1}-2$ to get

$$
\frac{\left|f(I)-l\left(f^{-q_{n+1}+q_{n}+1}(I)\right)\right|}{\left|f(I)-l\left(f^{-q_{n-1}+1}(I)\right)\right|} \leq\left(\left(R_{1} /|I|\right) \operatorname{dist}\left(f^{q_{n-1}}(I), I\right)\right)^{a_{n+1}-1} .
$$

The next inequality will relate estimates (13) and (11). RRI written for $\left\{r\left(f^{-q_{n}+1}(I)\right), f(I), l\left(f^{-q_{n+1}+q_{n}+1}(I)\right) ; q_{n+1}-1\right\}$ implies 


$$
\begin{gathered}
\frac{\left|f(I)-l\left(f^{-q_{n+1}+q_{n}+1}(I)\right)\right|}{\left|f(I)-r\left(f^{-q_{n}+1}(I)\right)\right|} \geq\left(1 / R_{1}\right) \frac{\left|f^{q_{n}}(I)-f^{q_{n+1}}(I)\right|}{\left|f^{q_{n+1}}(I)-f^{q_{n+1}-q_{n}}(I)\right|} \\
\geq \frac{|I| / R_{1}}{\left|l(I)-f^{q_{n+1}-q_{n}}(I)\right|} \geq\left(|I| / R_{1}\right) \operatorname{dist}\left(f^{q_{n-1}}(I), I\right)^{-1}
\end{gathered}
$$

Combining the inequalities (11), (13) and (14) we obtain

$$
\frac{\left|f^{-q_{n}}(I)\right|}{\left|r\left(f^{-q_{n}}(I)\right)-l(I)\right|} \geq K_{12}\left(\left(R_{1} /|I|\right) \operatorname{dist}\left(f^{q_{n-1}}(I), I\right)\right)^{-a_{n+1} / \ell}
$$

and consequently

$$
\begin{aligned}
\operatorname{dist}\left(f^{q_{n+1}}\right. & (I), I) \\
& \leq K_{13}\left(\left(R_{1} /|I|\right) \operatorname{dist}\left(f^{q_{n-1}}(I), I\right)\right)^{a_{n+1} / \ell} \operatorname{dist}\left(f^{q_{n-1}}(I), I\right) \\
& \leq\left(K_{14} \operatorname{dist}\left(f^{q_{n-1}}(I), I\right)\right)^{1+a_{n+1} / \ell} .
\end{aligned}
$$

If $a_{n+1}=1$ then 15 and $(16)$ follow directly from (14) and 111 .

Let $1 \leq m \leq n$ be even numbers. Then

$$
\gamma(n, m)=\prod_{m / 2<i \leq n / 2}\left(1+a_{2 i+1} / \ell\right)
$$

The recursive estimate 16 implies that

$$
\operatorname{dist}\left(f^{q_{n+1}}(I), I\right) \leq K_{14}^{\gamma(n, n-2)+\gamma(n, n-4)+\cdots+\gamma(n, m)} \operatorname{dist}\left(f^{q_{m+1}}(I), I\right)^{\gamma(n, m)} .
$$

Observe that

$$
\begin{aligned}
\sum_{i=0}^{(n-m) / 2} \gamma(n, m+2 i) & =\gamma(n, m) \sum_{i=0}^{(n-m) / 2} \gamma(m+2 i, m)^{-1} \\
& \leq \gamma(n, m) \sum_{i=0}^{\infty}(1+1 / \ell)^{-i} \leq(\ell+1) \cdot \gamma(n, m)
\end{aligned}
$$

Consequently,

$$
\operatorname{dist}\left(f^{q_{n+1}}(I), I\right) \leq\left(K_{15} \operatorname{dist}\left(f^{q_{m+1}}(I), I\right)\right)^{\gamma(n, m)} .
$$

By Lemma 3.1, there is a uniform constant $0<\lambda_{1}<1$ such that for every $n \in \mathbb{N}, \operatorname{dist}\left(f^{q_{n}}(I), I\right)<\lambda_{1}^{n}$. Choose $n_{0}$ even so that $K_{15} \operatorname{dist}\left(f^{q_{n_{0}+1}}(I), I\right) \leq$ $1 / 2$. The constant $n_{0}$ is uniform and, from (17), if $n \geq n_{0}$ is even then

$$
\operatorname{dist}\left(f^{q_{n+1}}(I), I\right) \leq(1 / 2)^{\gamma\left(n, n_{0}\right)} .
$$

Let $\lambda_{2}=(1 / 2)^{1 / \gamma\left(n_{0}\right)}$. Using 17 and 18 , we see that for every $n \geq n_{0}$ even,

$$
\operatorname{dist}\left(f^{q_{n+1}}(I), I\right) \leq(1 / 2)^{\gamma\left(n, n_{0}\right)}=(1 / 2)^{\gamma(n, 1) / \gamma\left(n_{0}, 1\right)}=\lambda_{2}^{\gamma(n)}=\lambda_{2}^{\gamma(n+1)} .
$$

The constant $\lambda_{2}$ is not uniform and it depends on the rotation number. Since $\operatorname{dist}\left(f^{q_{n+1}}(I), I\right)$ are all smaller than 1 , we obtain the first formula of $\mathbf{F B L}$ 
for all $n$ odd. The second formula of FBL for $n$ even follows from the first for $n$ odd and (15).

Let $N \geq n_{0}+1$ be an odd number chosen so that $K_{15}\left(\operatorname{dist}\left(f^{q_{N}}(I), I\right)\right)^{1 / 2}$ $<1$. The explicit formula for $\lambda$ stated in FBL can be derived from (17). Let $A=1 / 2$ and denote $\theta=\left(\operatorname{dist}\left(f^{q_{N}}(I), I\right)\right)^{A / \gamma(N)}$. If $n+1 \geq N$ then the inequality (17) with $m+1:=N$ implies that $\operatorname{dist}\left(f^{q_{n+1}}(I), I\right)$ is bounded by

$$
\left(\operatorname{dist}\left(f^{q_{N}}(I), I\right)\right)^{\gamma(n, N-1) / 2}=\left(\operatorname{dist}\left(f^{q_{N}}(I), I\right)\right)^{\gamma(n, N) / 2}=\theta^{\gamma(n+1)} .
$$

4.2. Proof of FBL when $f^{-q_{n}}(I)$ is to the right of $I$. The claims of FBL in this case follow easily from the estimates of the last subsection. Indeed, note that $f^{q_{n+1}}(I)$ lies between $f^{-q_{n+2}}(I)$ and $f^{-q_{n+2}+q_{n+1}}(I)$. Therefore,

$$
\operatorname{dist}\left(f^{q_{n+1}}(I), I\right) \leq\left|r(I)-l\left(f^{-q_{n+2}+q_{n+1}}(I)\right)\right| .
$$

Apply RRI for

$$
\left\{r(I), l\left(f^{-q_{n}-i q_{n+1}}(I)\right), r\left(f^{-q_{n}-i q_{n+1}}(I)\right) ; q_{n}+i q_{n+1}\right\},
$$

where $0 \leq i \leq a_{n+2}-1$, and a simple algebra to get

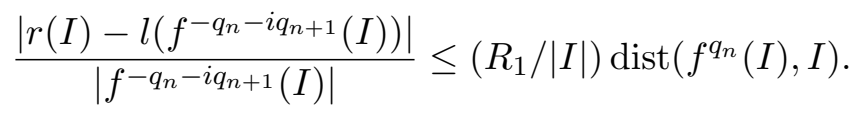

Multiplying through (21) for $0 \leq i \leq a_{n+2}-1$, we obtain

$$
\begin{aligned}
\frac{\left|r(I)-l\left(f^{-q_{n+2}+q_{n+1}}(I)\right)\right|}{\left|r(I)-r\left(f^{-q_{n}}(I)\right)\right|} & \leq \prod_{i=0}^{a_{n+2}-1} \frac{\left|r(I)-l\left(f^{-q_{n}-i q_{n+1}}(I)\right)\right|}{\mid r(I)-r\left(f^{\left.-q_{n}-i q_{n+1}(I)\right) \mid}\right.} \\
& \leq\left(\left(R_{1} /|I|\right) \operatorname{dist}\left(f^{q_{n}}(I), I\right)\right)^{a_{n+2}} .
\end{aligned}
$$

The inequality 200 and $\left|r(I)-r\left(f^{-q_{n}}(I)\right)\right| \leq \operatorname{dist}\left(f^{q_{n-1}}(I), I\right)$ allow us to write (21) in the recursive form

$$
\operatorname{dist}\left(f^{q_{n+1}}(I), I\right) \leq \operatorname{dist}\left(f^{q_{n-1}}(I), I\right)\left(\left(R_{1} /|I|\right) \operatorname{dist}\left(f^{q_{n}}(I), I\right)\right)^{a_{n+2}} .
$$

We recall that $n_{0}$ is a uniform constant defined in the discussion leading to the estimate (18). Suppose that $R_{1} /|I|>2$ and choose $n_{1}>n_{0}$ so that $\left(|I| / R_{1}\right)^{2}>(1 / 2)^{\gamma\left(n_{1}, n_{0}\right)}$. The constant $n_{1}$ is uniform since it can be defined as a number for which

$$
(2 / \log 2) \log \left(R_{1} /|I|\right)<(1+1 / \ell)^{\left(n_{1}-n_{0}\right) / 2} .
$$

Further, for every $1 \leq m \leq n$,

$$
a_{n+2} \cdot \gamma(n, m) \geq \gamma(n+1, m) /(\ell+1) .
$$


We use the estimate (19). Consequently, for every $n \geq n_{1}$ there exists $\lambda_{3} \in$ $(0,1)$ such that

$$
\begin{aligned}
\operatorname{dist}\left(f^{q_{n+1}}(I), I\right) & \leq\left(\left(R_{1} /|I|\right)(1 / 2)^{\gamma\left(n, n_{0}\right)}\right)^{a_{n+2}} \\
& \leq(1 / 2)^{\left(\gamma\left(n, n_{0}\right)-(1 / 2) \gamma\left(n_{1}, n_{0}\right)\right) a_{n+2}} \\
& \leq(1 / 2)^{a_{n+2} \cdot \gamma\left(n, n_{1}\right)} \\
& \leq(1 / \sqrt[\ell+1]{2})^{\gamma\left(n+1, n_{1}\right)} \leq \lambda_{3}^{\gamma(n+1)}
\end{aligned}
$$

This proves the first estimate of FBL. The constant $\lambda_{3}$ is not uniform as it is defined by

$$
\lambda_{3}=(1 / 2)^{1 /\left((\ell+1) \gamma\left(n_{1}\right)\right)} .
$$

The second inequality of FBL follows directly from the first and RRI written for $\left\{r(I), l\left(f^{-q_{n}}(I)\right), r\left(f^{-q_{n}}(I)\right) ; q_{n-1}\right\}$. Indeed,

$$
\frac{\left|f^{-q_{n}}(I)\right|}{\left|r(I)-l\left(f^{-q_{n}}(I)\right)\right|} \geq\left(|I| / R_{1}\right)\left(\operatorname{dist}\left(f^{q_{n}}(I), I\right)\right)^{-1} .
$$

The explicit formula for $\lambda$ in the statement of FBL is a consequence of (23) combined with the estimates (24) and (17). Indeed, choose $N \geq n_{0}$ odd so that $\left(K_{15} R_{1} /|I|\right)\left(\operatorname{dist}\left(f^{q_{N}}(I), I\right)\right)^{1 / 2}<1$. We can also drop $\operatorname{dist}\left(f^{q_{n-1}}(I), I\right)$ from the inequality 23$)$. Set $A=1 /(2(\ell+1))$ and $\theta=\left(\operatorname{dist}\left(f^{q_{N}}(I), I\right)\right)^{A / \gamma(N)}$. Then, for all $n \geq N$ odd,

$$
\begin{aligned}
\operatorname{dist}\left(f^{q_{n+1}}(I), I\right) & \leq\left(\left(R_{1} /|I|\right) \operatorname{dist}\left(f^{q_{n}}(I), I\right)\right)^{a_{n+2}} \\
& \leq\left(K_{15}\left(R_{1} /|I|\right) \operatorname{dist}\left(f^{q_{N}}(I), I\right)\right)^{\gamma(n+1, N-1) /(\ell+1)} \\
& \leq\left(\operatorname{dist}\left(f^{q_{N}}(I), I\right)\right)^{\gamma(n+1, N) / 2(\ell+1)}=\theta^{\gamma(n+1)} .
\end{aligned}
$$

\subsection{Uniform version of FBL}

LEMmA 4.2. There is a uniform constant $n_{0} \in \mathbb{N}$ such that for every $n \geq n_{0}$,

$$
\operatorname{dist}\left(f^{q_{n}}(I), I\right) \leq 2^{-\gamma\left(n, n_{0}\right)}, \quad \frac{\left|f^{-q_{n}}(I)\right|}{\operatorname{dist}\left(f^{-q_{n}}(I), I\right)} \geq 2^{\gamma\left(n, n_{0}\right)} .
$$

Proof. The first inequality follows directly from the estimate 19 when $n$ is odd and from (23) when $n$ is even. The second inequality is a consequence of the first and the inequalities (15) and 26).

4.4. Proof of Theorem 1. The coefficients $a_{i}$ in the statement of FBL are positive integers.

\section{Analytic description of the dynamics}

5.1. Distortion. The crucial observation made in this subsection is that the logarithm of the total distortion of an iterate $f^{i}$ on an interval $J$ with the property that $J, \ldots, f^{i-1}(J)$ are contained in the holes of the $n$th partition 
$\mathcal{A}_{n}$ tends to 0 uniformly superexponentially provided $J, \ldots, f^{i-1}(J)$ do not intersect the hole $\Delta \in \mathcal{A}_{n}$ adjacent to the critical point $l(I)$. The explanation of this phenomenon is the following: by FBL the holes are substantially shorter than the neighboring preimages of the flat interval $I$ which separate the holes from the critical point.

Lemma 5.1 (Distortion Lemma). Let $J, \ldots, f^{i-1}(J), i \geq 1$, be a collection of intervals contained in the holes of $\mathcal{A}_{n}$ which do not intersect the hole $\Delta \in \mathcal{A}_{n}$ adjacent to $l(I)$. Then for any $z_{1}, z_{2} \in J$ the logarithm of the distortion

$$
\left|\log \frac{d f^{i}}{d x}\left(z_{1}\right)-\log \frac{d f^{i}}{d x}\left(z_{2}\right)\right|
$$

tends to 0 uniformly superexponentially when $n$ goes to infinity.

Proof. Let $z_{1}, z_{2}$ be in the same hole of $\mathcal{A}_{n}$ different from $\Delta$. By the Mean Value Theorem,

$$
\begin{aligned}
\mathcal{D} & :=\left|\log \frac{d f^{i}}{d x}\left(z_{1}\right)-\log \frac{d f^{i}}{d x}\left(z_{2}\right)\right| \leq \sum_{j=0}^{i-1}\left(\left|\frac{d^{2} f}{d x^{2}}\left(\xi_{j}\right)\right| /\left|\frac{d f}{d x}\left(\xi_{j}\right)\right|\right)\left|f^{j}(J)\right| \\
& \leq M_{0} \sum_{\square(n) \in \mathcal{A}_{n} \backslash\{\Delta\}} \frac{|\square(n)|}{\operatorname{dist}(\square(n), I)},
\end{aligned}
$$

where $\xi_{j} \in f^{j}(J)$. Let $I(\square(n)) \in \mathcal{A}_{n}$ be a preimage of the flat interval $I$ lying immediately to the right of $\square(n)$. By Fact 3.3, the ratio $|\square(n)| /|I(\square(n))|$ is bounded from above by

$$
\begin{aligned}
\left.\left(R_{1} /|I|\right) \operatorname{dist}\left(f^{q_{n}}(I), I\right)\right) & \text { if } n \text { is odd, } \\
\left.\left(R_{1} /|I|\right) \operatorname{dist}\left(f^{-q_{n}}(I), I\right)\right) & \text { if } n \text { is even. }
\end{aligned}
$$

If $n$ is odd then $\Delta$ coincides with $\left(r\left(f^{-q_{n+1}}(I)\right), l(I)\right)$ and $f^{q_{n}}(I)$ lies in the hole $\Delta_{1} \in \mathcal{A}_{n}$ between $f^{-q_{n+1}+q_{n}}(I)$ and $f^{-q_{n+1}}(I)$, i.e. $\Delta_{1}$ is to the left of $f^{-q_{n+1}}(I)$. By RRI written for $\left\{f^{q_{n}}(I), l\left(f^{-q_{n+1}}(I)\right), r\left(f^{-q_{n+1}}(I)\right) ; q_{n+1}\right\}$,

$$
\frac{\left|f^{q_{n}}(I)-l\left(f^{-q_{n+1}}(I)\right)\right|}{\left|f^{-q_{n+1}}(I)\right|} \leq\left(R_{1} /|I|\right) \operatorname{dist}\left(f^{q_{n+1}+q_{n}}(I), I\right) \leq R_{1} /|I|
$$

and

$$
\begin{aligned}
\operatorname{dist}\left(f^{q_{n}}(I), I\right) & =\operatorname{dist}\left(\Delta_{1}, I\right)+\left|f^{q_{n}}(I)-l\left(f^{-q_{n+1}}(I)\right)\right| \\
& \leq \operatorname{dist}\left(\Delta_{1}, I\right)\left(1+\frac{\left|f^{q_{n}}(I)-l\left(f^{-q_{n+1}}(I)\right)\right|}{\left|f^{-q_{n+1}}(I)\right|}\right) \\
& \leq \operatorname{dist}\left(\Delta_{1}, I\right)\left(1+R_{1} /|I|\right) .
\end{aligned}
$$


Hence, for $n$ odd,

$$
\begin{aligned}
\mathcal{D} & \leq M_{1} \sum_{\square(n) \in \mathcal{A}_{n} \backslash\{\Delta\}} \frac{|\square(n)|}{|I(\square(n))|} \frac{|I(\square(n))|}{\operatorname{dist}(\square(n), I)} \\
& \leq M_{2} \sum_{\square(n) \in \mathcal{A}_{n} \backslash\left\{\Delta, \Delta_{1}\right\}} \operatorname{dist}\left(\Delta_{1}, I\right)\left(1+\frac{|I(\square(n))|}{\operatorname{dist}(\square(n), I)}\right) \\
& \leq M_{3} \operatorname{dist}\left(\Delta_{1}, I\right) \int_{\operatorname{dist}\left(\Delta_{1}, I\right)}^{1} \frac{1}{x} d x \\
& =M_{3} \operatorname{dist}\left(\Delta_{1}, I\right)\left|\log \left(\operatorname{dist}\left(\Delta_{1}, I\right)\right)\right| .
\end{aligned}
$$

For $n$ even, $\Delta$ coincides with $\left(r\left(f^{-q_{n}}(I)\right), l(I)\right)$. Therefore,

$$
\frac{|\square(n)|}{|I(\square(n))|} \leq\left(R_{1} /|I|\right)|\Delta|
$$

and

$$
\begin{aligned}
\mathcal{D} & \leq M_{4} \sum_{\square(n) \in \mathcal{A}_{n} \backslash\{\Delta\}}|\Delta| \frac{|I(\square(n))|}{\operatorname{dist}(\square(n), I)} \\
& \leq M_{5}|\Delta| \int_{|\Delta|}^{1} \frac{1}{x} d x=M_{5}|\Delta||\log (|\Delta|)| .
\end{aligned}
$$

In view of FBL, the proof of Lemma 5.1 is complete.

In the next step we will estimate distortion on the holes of $\mathcal{A}_{n}$ adjacent to the flat interval $I$. We recall that the rotation number $\rho$ of $f$ has a continued fraction representation $\rho=\left[0, a_{1}, \ldots, a_{n}, \ldots\right]$.

Lemma 5.2. Denote by $\Delta \in \mathcal{A}_{n}$ the hole adjacent to I from the left and by $\Delta^{\prime}$ the whole adjacent to I from the right.

- For every $n \in \mathbb{N}$ even and every $0 \leq k<q_{n+1}$, the logarithm of the distortion of $f^{k}$ on $f(\Delta)$ tends uniformly and superexponentially to 0.

- For every $n \in \mathbb{N}$ odd and every $0 \leq i<a_{n+1}$, the logarithm of the distortion of $f^{q_{n}-1}$ on $f^{i q_{n}+1}\left(\Delta^{\prime}\right)$ tends uniformly and superexponentially to 0 .

Proof. For $n$ even, $\Delta=\square_{0}^{n}$. Observe that the intervals $\left\{f^{i}(\Delta): 0 \leq\right.$ $\left.i<q_{n+1}\right\}$ and $\left\{f^{-j}(I): 0<j \leq q_{n}\right\}$ are pairwise disjoint. This means that the intervals $\left\{f^{i}(\Delta): 0 \leq i<q_{n+1}\right\}$ are contained in the holes of $\mathcal{A}_{n-2}$ as $q_{n-1}+q_{n-2} \leq q_{n}$. Consider now those intervals of $\left\{f^{i}(\Delta): 0 \leq i<q_{n+1}\right\}$ which lie in the hole $\Delta_{1}:=\square_{0}^{n-2} \in \mathcal{A}_{n-2}$ adjacent to $l(I)$. Every such $f^{i}(\Delta)$ is disjoint from $\left\{f^{-q_{n-2}-m q_{n-1}}(I): 0 \leq m \leq a_{n}\right\}$ and thus it is contained in the union of the holes of $\mathcal{A}_{n-1} \backslash\{\Delta\}$. 
Denote by $\mathcal{B}_{n-1}$ the set of all holes of $\mathcal{A}_{n-1}$ contained in $\Delta_{1} \backslash \Delta$. As in the proof of Lemma 5.1 (see 27 ), for every $z_{1}, z_{2} \in f(\Delta)$ we have

$$
\begin{aligned}
\mathcal{D} & :=\left|\log \frac{d f^{k}}{d x}\left(z_{1}\right)-\log \frac{d f^{k}}{d x}\left(z_{2}\right)\right| \\
& \leq \sum_{j=0}^{k-1}\left(\left|\frac{d^{2} f}{d x^{2}}\left(\xi_{j}\right)\right| /\left|\frac{d f}{d x}\left(\xi_{j}\right)\right|\right)\left|f^{j+1}(\Delta)\right| \\
& \leq M_{6}\left(\sum_{\square \in A_{n-2} \backslash\left\{\Delta_{1}\right\}} \frac{|\square|}{\operatorname{dist}(\square, I)}+\sum_{\square \in \mathcal{B}_{n-1}} \frac{|\square|}{\operatorname{dist}(\square, I)}\right),
\end{aligned}
$$

where $\xi_{j} \in f^{j+1}(\Delta)$. Since $\mathcal{B}_{n-1} \subset \mathcal{A}_{n-1} \backslash\{\Delta\}$, we apply the estimates 31 and $(32)$ to conclude the proof.

For $n$ odd, $\Delta^{\prime}=\square_{0}^{n}$. Fix $0 \leq i<a_{n+1}$ and observe that the intervals $\left\{f^{i q_{n}+j}\left(\Delta^{\prime}\right): 1 \leq j<q_{n}\right\}$ are pairwise disjoint and contained in the union of the holes of $\mathcal{A}_{n-1} \backslash\left\{\square_{0}^{n-1}\right\}$. The claim follows by Lemma 5.1 .

We conclude this section with a corollary that the graph of $f^{q_{n+1}}$ for $n$ even and large in "the small scale" is similar to the graph of $x^{\ell}$ up to an affine change of coordinates.

COROLlaRY 5.1. There are uniform constants $n_{0}, M_{7}$ such that for every $n \geq n_{0}$ even and any two points $z_{1}, z_{2} \in \square_{i}^{n}, 0 \leq i<q_{n+1}$,

$$
\begin{aligned}
& \frac{1}{M_{7}}\left(\frac{\left|z_{1}-r\left(\square_{i}^{n}\right)\right|}{\left|z_{2}-r\left(\square_{i}^{n}\right)\right|}\right)^{\ell-1} \leq \frac{d f^{q_{n+1}}}{d x}\left(z_{1}\right) / \frac{d f^{q_{n+1}}}{d x}\left(z_{2}\right) \leq M_{7}\left(\frac{\left|z_{1}-r\left(\square_{i}^{n}\right)\right|}{\left|z_{2}-r\left(\square_{i}^{n}\right)\right|}\right)^{\ell-1} . \\
& \quad \text { Proof. Combine the estimates of Lemma 5.1, Lemma 5.2, and Fact 3.1. }
\end{aligned}
$$

5.2. Miscelaneous estimates. Now, we are in a position to show that the holes of the $n$th partition are very small not only in comparison to the preimages of $I$ which lie immediately to their right (see FBL) but also to their left.

LEMmA 5.3. There are uniform constants $n_{0}, M_{8}$ such that for every $n \geq n_{0}$ even and every $0 \leq i<q_{n+1}$,

$$
\frac{\left|f^{-i-q_{n}}(I)\right|}{\left|\square_{i}^{n}\right|} \geq\left(M_{8} \operatorname{dist}\left(f^{q_{n-1}}(I), I\right)\right)^{-a_{n+1} / \ell} .
$$

Proof. By RRI and the uniform version of FBL, the interval $f^{-i-q_{n}}(I)$ is very small in comparison to $f^{-i}(I)$. Hence, the ratio $\left|f^{-q_{n}-i}(I)\right| /\left|\square_{i}^{n}\right|$ is greater than a uniform constant multiple of $\left|f^{-q_{n}}(I)\right| /\left|\square_{0}^{n}\right|$. The lemma follows by the estimate 15 .

We will show that the map $f^{q_{n+1}}(z)$ is expanding with a large derivative provided $z \in \square_{i}^{n}$ is not too close to $l\left(\square_{i}^{n}\right)$. 
Lemma 5.4. There are uniform constants $n_{0}, M_{9}$ with the following property.

- For every $z \in \square_{i}^{n}, 0 \leq i \leq q_{n+1}$, and $n \geq n_{0}$ even,

$$
\frac{d f^{q_{n+1}}}{d x}(z) \geq\left(M_{9} \operatorname{dist}\left(f^{q_{n-1}}(I), I\right)\right)^{-a_{n} / \ell}\left(\frac{\left|z-r\left(\square_{i}^{n}\right)\right|}{\left|\square_{i}^{n}\right|}\right)^{\ell-1} .
$$

- For every $z \in\left(l\left(\square_{i}^{n}\right), l\left(f^{-q_{n+1}-q_{n}-i}(I)\right), 0 \leq i<q_{n+1}\right.$, and $n \geq n_{0}$ odd,

$$
\frac{d f^{q_{n+1}}}{d x}(z) \geq \frac{M_{9}}{\operatorname{dist}\left(f^{q_{n}}(I), I\right)} .
$$

Proof. Assume that $n$ is even. Note that $f^{-q_{n}-i}(I) \subset f^{q_{n+1}}\left(\square_{i}^{n}\right)$. By the Mean Value Theorem and the Convexity Lemma, there exists $\xi \in \square_{i}^{n}$ such that

$$
\frac{d f^{q_{n+1}}}{d x}\left(l\left(\square_{i}^{n}\right)\right) \geq \frac{d f^{q_{n+1}}}{d x}(\xi) \geq \frac{\left|f^{-i-q_{n}}(I)\right|}{\left|\square_{i}^{n}\right|} .
$$

Substitute the above inequality in the formula of Corollary 5.1 with $z_{1}$ and $z_{2}$ replaced, respectively, by $z$ and $l\left(\square_{i}^{n}\right)$. The estimate follows by Lemma 5.3 .

Assume now that $n$ is odd. Since $f^{-q_{n+1}-q_{n}-i}(I) \subset \square_{i}^{n}$, there is $\xi \in$ $f^{-q_{n+1}-q_{n}-i}(I)$ such that

$$
\frac{d f^{q_{n+1}}}{d x}(\xi) \geq \frac{\left|f^{-i-q_{n}}(I)\right|}{\left|\square_{i}^{n}\right|} .
$$

By Lemma 5.1, the graph of $f^{q_{n+1}}$ on $\left(l\left(\square_{i}^{n}\right), l\left(f^{-q_{n+1}-q_{n}-i}(I)\right)\right.$ is almost linear. Hence, for every $z$ from that interval,

$$
\frac{d f^{q_{n+1}}}{d x}(z)>M_{10} \frac{\left|f^{-i-q_{n}}(I)\right|}{\left|\square_{i}^{n}\right|} .
$$

The second estimate of the lemma follows from 26 .

5.3. Nonlinearity. The one-form $\mathcal{N} f=\left(f^{\prime \prime} / f^{\prime}\right) d x$, called the nonlinearity of $f$, measures the infinitesimal change of the distortion of $f$ at the point $x$. We will use this quantity to get convexity estimates of the second derivative of $f^{q_{n}}$.

FACT 5.1. There exist uniform positive constants $H_{1}$ and $H_{2}$ with the following properties. For every $x$ outside the flat interval $I$, we have

$$
|\mathcal{N} f(x)| \operatorname{dist}(x, I) \leq H_{1} .
$$

Additionally, if $x$ is close enough to $l(I)$ then

$$
\mathcal{N} f(x) \operatorname{dist}(x, I) \leq-H_{2} \text {. }
$$

Let $I \subset \mathbb{R}$ be an interval and $f: I \rightarrow \mathbb{R}$ be a $C^{2}$ function. We say that $f$ is upper convex if $f^{\prime \prime}(x) \leq 0$ for every $x \in I$. 
The Convexity Lemma states that $f^{q_{n+1}}$ on $\square_{i}^{n}$ is upper convex for $n$ even and large.

Lemma 5.5 (Convexity Lemma). There are uniform constants $n_{0}, H>0$ with the following property. If $n \geq n_{0}$ is even then for every $\square_{i}^{n}$ of the partition $\mathcal{A}_{n}$,

$$
\left|\square_{i}^{n}\right| \mathcal{N} f^{q_{n+1}}(x) \leq-H .
$$

In particular, the graph of $f^{q_{n+1}}$ restricted to $\square_{i}^{n}$ is upper convex.

Proof. We choose $n_{0}$ so that for every $n$ even and greater than $n_{0}$, the second derivative of $f$ is negative on $\square_{0}^{n}$.

By Lemma 5.1 , there is $H_{4}$ such that for every $z \in \square_{i}^{n}$,

$$
\frac{d f^{i}}{d x}(x) \geq H_{4} \frac{\left|\square_{0}^{n}\right|}{\left|\square_{i}^{n}\right|} .
$$

There is a uniform constant $H_{5}$ such that $\mathcal{N}^{+} f:=\max (0, \mathcal{N} f) \leq H_{5}$. After these preparations we are ready to estimate the rescaled nonlinearity $\left|\square_{i}^{n}\right| \mathcal{N} f^{q_{n+1}}(x)$ on the interval $\square_{i}^{n}$. By the chain rule, Lemmas 5.1, 5.2, and Fact 5.1 .

$$
\begin{aligned}
\left|\square_{i}^{n}\right| \mathcal{N} f^{q_{n+1}}(x) & =\left|\square_{i}^{n}\right| \sum_{j=0}^{q_{n+1}-1} \mathcal{N} f\left(f^{j}(x)\right) \frac{d f^{j}}{d x}(x) \\
& \leq H_{6}\left|\square_{0}^{n}\right| \mathcal{N} f\left(f^{i}(x)\right)+H_{7} \sum_{j=0, j \neq i}^{q_{n+1}-1} \mathcal{N}^{+} f\left(f^{j}(x)\right) \frac{d f^{j}}{d x}(x)\left|\square_{i}^{n}\right| \\
& \leq-H_{8} \frac{\left|\square_{0}^{n}\right|}{\operatorname{dist}\left(f^{i}(x), I\right)}+H_{9} \sum_{j=0, j \neq i}^{q_{n+1}-1}\left|f^{j}\left(\square_{i}^{n}\right)\right| \\
& \leq-H_{8}+H_{9} \sum_{\square \in \mathcal{A}_{n-1} \cup \mathcal{A}_{n-2}}|\square| \leq-H_{10},
\end{aligned}
$$

as the total length of the holes of $\mathcal{A}_{n-1}$ and $\mathcal{A}_{n-2}$ tends to 0 uniformly superexponentially. The estimate

$$
\frac{d f^{j}}{d x}(x)\left|\square_{i}^{n}\right| \leq H_{10}\left|f^{j}\left(\square_{i}^{n}\right)\right| \quad \text { for every } 0 \leq j<q_{n+1}
$$

also uses Fact 3.1

6. Scaling rules-lower bounds. The proof of SBL falls into two parts.

6.1. Proof of SBL when $f^{-q_{n}}(I)$ is to the left of $I$. Throughout the proof of SBL we will usually use the letter $A$ with suitable subscripts to denote subsequent uniform constants appearing in the estimates. By Lemma 4.1 . 


$$
\operatorname{dist}\left(f^{q_{n+1}}(I), I\right) \geq\left|f^{-q_{n+2}}(I)\right| \geq K_{6}\left|r(I)-l\left(f^{-q_{n+1}}(I)\right)\right|^{1 / \ell} .
$$

Since the first derivative of $f$ is bounded,

$$
\left|r(I)-l\left(f^{-q_{n+1}}(I)\right)\right| \geq A_{0}\left|f(I)-l\left(f^{-q_{n+1}+1}(I)\right)\right| .
$$

By Fact 3.1 .

$$
\left|r\left(f^{-q_{n}+1}(I)\right)-f(I)\right| \geq K_{1}\left|r\left(f^{-q_{n}}(I)\right)-l(I)\right|^{\ell} .
$$

Write RRI for $\left\{r\left(f^{-q_{n}+1}(I)\right), f(I), l\left(f^{-q_{n+1}+1}(I)\right) ; q_{n+1}-1\right\}$ and then combine it with (35), (36) to obtain

$$
\begin{aligned}
\frac{\left|r(I)-l\left(f^{-q_{n+1}}(I)\right)\right|}{\left|r\left(f^{-q_{n}}(I)\right)-l(I)\right|^{l}} & \geq \frac{A_{0} K_{1}}{R_{1}} \frac{\left|f^{q_{n+1}}(I)-l(I)\right|}{\left|f^{-q_{n}+q_{n+1}}(I)-f^{q_{n+1}}(I)\right|} \\
& \geq A_{1} \frac{\left|f^{q_{n+1}}(I)-l(I)\right|}{\left|l\left(f^{-q_{n}}(I)\right)-l(I)\right|},
\end{aligned}
$$

the last inequality being a consequence of the fact that $f^{-q_{n}+q_{n+1}}(I) \in \square_{q_{n}}^{n}$ (Fact 3.3 and FBL imply that $\left|f^{-q_{n}+q_{n+1}}(I)-l\left(f^{-q_{n}}(I)\right)\right|$ is much smaller than $\left.\left|f^{-q_{n}}(I)\right|\right)$.

Substituting (34) into (37) and then taking the $\ell$ th root of both sides, we get

$$
\frac{\left|f^{q_{n+1}}(I)-l(I)\right|}{\left|r\left(f^{-q_{n}}(I)\right)-l(I)\right|} \geq A_{2} \frac{\left|f^{q_{n+1}}(I)-l(I)\right|^{1 / \ell}}{\left|l\left(f^{-q_{n}}(I)\right)-l(I)\right|^{1 / \ell}} .
$$

Let $\left.b_{n}=\mid f^{q_{n}}(I)\right)-l(I) \mid$. By FBL (see also Lemma 4.2), $b_{n-1}$ and $\left|l\left(f^{-q_{n}}(I)\right)-l(I)\right|$ are equal up to a superexponentially small error when $n$ goes to infinity. Also, $\left|r\left(f^{-q_{n}}(I)\right)-l(I)\right| \geq b_{n+1}$. Therefore, (38) becomes

$$
\left(\frac{b_{n+1}}{b_{n-1}}\right)^{(\ell-1) / \ell} \geq A_{3} \frac{\left|r\left(f^{-q_{n}}(I)\right)-l(I)\right|}{\left|l\left(f^{-q_{n}}(I)\right)-l(I)\right|} .
$$

Applying RRI for $\left\{l\left(f^{-q_{n}+1}(I)\right), r\left(f^{-q_{n}+1}(I)\right), f(I) ; q_{n}-1\right\}$ and using the fact that $\left|\square_{0}^{n}\right|$ is superexponentially smaller than $\left|f^{-q_{n}}(I)\right|$, we get

$$
\left(\frac{\left|r\left(f^{-q_{n}}(I)\right)-l(I)\right|}{\left|l\left(f^{-q_{n}}(I)\right)-l(I)\right|}\right)^{\ell} \geq A_{4} b_{n}
$$

and consequently

$$
\left(\frac{b_{n+1}}{b_{n-1}}\right)^{\ell-1} \geq A_{5} b_{n}
$$

Our objective now is to find a recurrence formula for $b_{n+1} / b_{n-1}$. To this end we prove the following lemma.

Lemma 6.1. For every $2 \leq i \leq a_{n+1}$,

$$
\frac{\left|r(I)-f^{(i-1) q_{n}}(I)\right|}{\left|r(I)-f^{i q_{n}}(I)\right|} \geq A_{6}\left|r(I)-l\left(f^{-q_{n-1}}(I)\right)\right| .
$$


Proof. By Lemmas 4.1 and 3.1 .

$$
\left|f(I)-l\left(f^{-q_{n-1}+1}(I)\right)\right| \leq A_{7}\left|f(I)-l\left(f^{-q_{n}+1}(I)\right)\right| .
$$

By 40 and RRI written for $\left\{l\left(f^{-q_{n}+1}(I)\right), f(I), f^{(i-1) q_{n}+1}(I) ; q_{n}-1\right\}$,

$$
\begin{aligned}
\frac{\left|r(I)-f^{(i-1) q_{n}}(I)\right|}{\left|r(I)-l\left(f^{-q_{n-1}}(I)\right)\right|} & \geq A_{8} \frac{\left|f(I)-f^{(i-1) q_{n}+1}(I)\right|}{\left|f(I)-l\left(f^{-q_{n}+1}(I)\right)\right|} \\
& \geq A_{9} \frac{\left|f^{q_{n}}(I)-f^{i q_{n}}(I)\right|}{\left.\mid f^{q_{n}}(I)-l(I)\right) \mid} \\
& \geq A_{10}\left|f^{q_{n}}(I)-f^{i q_{n}}(I)\right| .
\end{aligned}
$$

Since $\left(f^{q_{n}}(I), f^{i q_{n}}(I)\right) \supset f^{-q_{n+1}+q_{n}}(I)$, the lemma follows by Fact 3.3 .

Multiplying through the inequalities of Lemma 6.1 for $2 \leq i \leq a_{n+1}$, we obtain

$$
b_{n} \geq\left|r(I)-f^{a_{n+1} q_{n}}(I)\right|\left(A_{10}\left|r(I)-l\left(f^{-q_{n-1}}(I)\right)\right|\right)^{a_{n+1}-1} .
$$

Note that $f^{-q_{n}-q_{n-1}}(I) \subset\left(r(I), f^{a_{n} q_{n}}(I)\right)$. Hence, Lemma 5.3 implies that the intervals $\left(r(I), f^{a_{n} q_{n}}(I)\right)$ and $\left(r(I), l\left(f^{-q_{n-1}}(I)\right)\right)$ are of the same size up to a superexponentially small error. Hence,

$$
b_{n} \geq\left(A_{11}\left|r(I)-l\left(f^{-q_{n-1}}(I)\right)\right|\right)^{a_{n+1}} .
$$

The inequality (42) is also true for $a_{n+1}=1$ and follows directly from Lemma 5.3 and the inequality

$$
r\left(f^{-q_{n+1}}(I)\right)=r\left(f^{-q_{n}-q_{n-1}}(I)\right) \leq f^{q_{n}}(I) \leq l\left(f^{-q_{n-1}}(I)\right) .
$$

Indeed, $\left|f^{-q_{n-1}-q_{n}}(I)\right| \geq M_{8}\left|\square_{q_{n-1}}^{n}\right|=M_{8}\left|r\left(f^{-q_{n+1}}(I)\right)-l\left(f^{-q_{n-1}}(I)\right)\right|$ and hence $b_{n}$ and $\left|r(I)-l\left(f^{-q_{n-1}}(I)\right)\right|$ are uniformly comparable.

The inequality

$$
\begin{aligned}
\frac{\left|r(I)-l\left(f^{-q_{n-1}}(I)\right)\right|}{b_{n-1}^{\ell}} & \geq A_{12} b_{n-1}\left(\operatorname{dist}\left(f^{2 q_{n-1}}(I), f^{q_{n-1}}(I)\right)\right)^{-1} \\
& \geq A_{12} \frac{b_{n-1}}{b_{n-3}}
\end{aligned}
$$

is a simple consequence of Fact 3.1 and $\mathbf{R R I}$ written for $\left\{f^{q_{n-1}+1}(I), f(I)\right.$, $\left.l\left(f^{-q_{n-1}}(I)\right) ; q_{n-1}-1\right\}$. By FBL, the quantity $A_{12} / b_{n-3}$ tends uniformly to $\infty$. The inequality 43 becomes

$$
\left|r(I)-l\left(f^{-q_{n-1}}(I)\right)\right| \geq A_{13}\left(b_{n-1}\right)^{\ell+1},
$$

where $A_{13}$ can be made arbitrarily large by increasing $n$.

Substituting (44) into 42, we obtain

$$
b_{n} \geq\left(A_{14} b_{n-1}\right)^{(\ell+1) a_{n+1}},
$$

which together with $(39)$ gives the recurrence formula

$$
b_{n+1} \geq\left(A_{15} b_{n-1}\right)^{1+a_{n+1} \frac{\ell+1}{\ell-1}} .
$$


There is a uniform constant $N$ such that $A_{14}, A_{15}>1$ for every $n \geq N$. We may assume that $N$ is odd. Let $\omega=b_{N}^{1 / \bar{\gamma}(N)}$. The recurrence formula yields for every $n+1 \geq N$,

$$
b_{n+1} \geq A_{15} b_{N}^{\bar{\gamma}_{N}^{(n+1) / \bar{\gamma}(N)}}=A_{15} \omega^{\bar{\gamma}(n+1)} \geq \omega^{\bar{\gamma}(n+1)} .
$$

This proves the first formula of SBL for $n$ odd. The second formula of SBL for $n$ even is an immediate consequence of the first and the inequalities (39) and (45) if only $A_{14}$ is chosen large enough.

6.2. Proof of SBL when $f^{-q_{n}}$ is to the right of $I$. The first inequality follows directly from (45) and (46). We adjust the indices in these formulas to reflect the fact that $n$ is assumed to be odd in Subsection 6.2. Since $A_{14}>1$, there is a uniform constant $A_{16}$ such that for every $n \geq N$ odd,

$$
b_{n+1} \geq A_{14} b_{n}^{(\ell+1) a_{n+2}} \geq A_{14} \omega^{(\ell+1) a_{n+2} \bar{\gamma}(n)} \geq \omega^{A_{16} \bar{\gamma}(n+1)} .
$$

The second inequality can be obtained from CRI written for

$$
\left\{r(I), l\left(f^{-q_{n}}(I)\right), r\left(f^{-q_{n}}(I)\right), l\left(f^{-q_{n}-q_{n-1}}(I)\right) ; q_{n}\right\} .
$$

Indeed, using $b_{n} \geq A_{15} \omega^{\bar{\gamma}(n)}$ and $b_{n-1} \geq \omega^{A_{16} \bar{\gamma}(n-1)}$, we deduce that for every $n \geq N$ odd

$$
\frac{\left|r(I)-l\left(f^{-q_{n}}(I)\right)\right|}{\left|f^{-q_{n}}(I)\right|} \geq A_{10} b_{n} b_{n-1} \geq\left(A_{10} A_{15}\right) \omega^{\bar{\gamma}(n)+A_{16} \bar{\gamma}(n-1)} \geq \omega^{A_{17} \bar{\gamma}(n)},
$$

where $A_{17}$ is a uniform constant and $A_{15}$ is chosen so that $A_{15} A_{10}>1$.

Acknowledgments. I would like to thank Feliks Przytycki for his support and encouragement to publish the paper. Leo Jonker helped with many discussions during my visit to the Queen's University at Kingston whose warm hospitality is acknowledged. I am also grateful to Sylvain Crovisier for many helpful comments on the manuscript. Finally, I would like to thank the referee for carefully reading the manuscript and contributing numerous suggestions which greatly improved the exposition.

This research was partially supported by EU Research Training Network CODY.

\section{References}

[1] J. Graczyk, L. B. Jonker, G. Świątek, F. M. Tangerman and J. J. P. Veerman, Differentiable circle maps with a flat interval, Comm. Math. Phys. 173 (1995), 599622 .

[2] R. Ito, Rotation sets are closed, Math. Proc. Cambridge Philos. Soc. 89 (1981), 107111.

[3] M. Misiurewicz, Rotation interval for a class of maps of the real line into itself, Ergodic Theory Dynam. Systems 6 (1986), 117-132. 
[4] G. Swiątek, Rational rotation numbers for maps of the circle, Comm. Math. Phys. 119 (1988), 109-128.

[5] J. J. P. Veerman and F. M. Tangerman, Scalings in circle maps I, ibid. 134 (1990), $89-107$.

Jacek Graczyk

Université de Paris-Sud, Mathématique

91405 Orsay, France

E-mail: jacek.graczyk@math.u-psud.fr

Received 16 October 2009;

in revised form 14 February 2010 